\title{
Performance of Ge-Doped Optical Fiber as a Thermoluminescent Dosimeter
}

\author{
M. Benabdesselam, Member, IEEE, F. Mady, Member, IEEE, S. Girard, Senior Member, IEEE, \\ Y. Mebrouk, Student Member, IEEE, J. B. Duchez, Student Member, IEEE, M. Gaillardin, Member, IEEE, and \\ P. Paillet, Senior Member, IEEE
}

\begin{abstract}
We have investigated the thermoluminescent response of three Germanium-doped silica-based optical fibers obtained by varying the drawing parameters from a unique preform. We compared under X-ray irradiation, their dosimetric properties to those of two widely used commercial dosimeters based on different technologies. Then, we investigated the potential of these optical fibers to monitor gamma rays at different doses and dose-rates, and to different fluences of particles $(0.8$ and $14 \mathrm{MeV}$ neutrons and $63 \mathrm{MeV}$ protons). Our results show that the thermoluminescence response of the Ge-doped optical fibers depends linearly on the direct ionizing dose (gamma-, X-rays) or on the indirect ionizing dose (protons, neutrons). As a consequence, this class of fibers is an excellent candidate for passive dosimetry in various fields from medical applications to high-energy physics.
\end{abstract}

Index Terms-Dosimeters, dosimetry, gamma rays, Germanium, irradiation, neutrons, optical fiber, protons, radiation therapy, thermoluminescence.

\section{INTRODUCTION}

$\mathbf{R}$ ADIATION effects in both doped and undoped optical fibers have been investigated for a long time [1]-[4]. Most of these studies have been devoted to identify suitable radiation-tolerant optical fibers for integration intended for communication links, diagnostics, control-command applications, and fiber sensors. However, during the last decade, a more limited number of investigations on the potential of commercially available silica based optical fibers for ionizing radiation dosimetry have been carried out using the thermally stimulated luminescence (TSL) technique [5]-[7]. In these investigations, the authors were only interested in knowing the TSL sensitivity of the fibers to both photons and electrons irradiations. However, the performed investigations are only limited to low absorbed doses (10 krad) or to current representative value of dose levels in medical applications.

Manuscript received July 03, 2013; revised September 16, 2013; accepted September 29, 2013. Date of publication December 03, 2013; date of current version December 11, 2013.

M. Benabdesselam, F. Mady, Y. Mebrouk, and J.B. Duchez are with the Université Nice Sophia Antipolis, CNRS, Laboratoire Physique de la Matière Condensée, UMR 7336, F-06108, Nice cedex 2, France (e-mail: ben@unice.fr; Franck.Mady@unice.fr; Yasmine.Mebrouk@unice.fr; jbduchez@unice.fr).

S. Girard is with the Laboratoire Hubert Curien, UMR CNRS 5516, Université de Saint-Etienne, F-42000 Saint-Etienne, France (email: sylvain.girard@univ-st-etienne.fr).

M. Gaillardin and P. Paillet are with the CEA, DIF, F-91680 Bruyères-le Châtel, France (e-mail: marc.gaillardin@cea.fr; philippe.paillet@cea.fr).

Color versions of one or more of the figures in this paper are available online at http://ieeexplore.ieee.org.

Digital Object Identifier 10.1109/TNS.2013.2284289
The aim of our work is twofold. First, to demonstrate the actual ability of a Germanium $(\mathrm{Ge})$ doped optical fiber to be used as a TSL-mode dosimeter and to confirm its applicability. Under this framework, it is interesting to explore the TSL response of such a dosimeter to photons $(\mathrm{X}, \gamma)$ within a dose range higher than $10 \mathrm{krad}$, at different dose rates and as well as its behavior to different fluences of protons and neutrons. Then, such TSL dosimetry efficiency can be extended for dose monitoring application in nuclear industry, especially in facilities where the fusion study, either by inertial or magnetic confinement, is performed or high-energy physics facilities.

After that, it is necessary to verify if the Ge-doped optical fiber based dosimeter, fits at least the main criteria required by such applications in terms of sensitivity and linearity of the response, reproducibility, storage stability and reliability by conducting a comparative study between the widely used commercial dosimeters based on different materials and the proposed Ge-doped optical fibers based dosimeters.

In addition, it is well known that the principle of TSL dosimetry is based on the use of the property of the TSL response involving deep traps that are thermally stable at room temperature (RT), where the increasing population corresponds to radiation-absorbed dose. In this technique, the deep level acts as "a memory cell" of the ionizing radiation dose. The trapping features such as the trap depth and the frequency factor of the involved point defect and its concentration in the TSL mechanism, among others, are the critical parameters that control the response of the TSL dosimeter (TLD). These trapping parameters are determined in the optical fibers within the scope where the traps are related to the radiation-induced point defects that are generated, either by ionization or displacement damage in the amorphous silica glass constituting the fiber core and cladding [8].

The second aim is to investigate the ability of Ge-doped optical fiber to provide useful information on the absorbed dose in various radiation environments ( $\gamma$, neutrons and protons).

Moreover, this study focuses on on-demand samples in which the full knowledge of their physicochemical properties, as well as their synthesis and drawing conditions, are being heavily investigated. Our objective is to further improve the performance of this promising TSL dosimeter with respect to the changes in its fabrication process.

\section{EXPERIMENTAL DETAILS}

\section{A. Studied Fibers and Dosimeters}

Multimode fibers GeD1, GeD2 and GeD3 (core and cladding diameters of 62.5 and $125 \mu \mathrm{m}$ respectively) used in this work 
TABLE I

Summary of the PhysicAl Characteristics of SAMPLes

\begin{tabular}{ccc}
\hline Dosimeter & Provider & $\begin{array}{c}\text { Form } \\
\text { Size }(\mathrm{mm}) \\
\end{array}$ \\
& & Mass $(\mathrm{mg})$ \\
\hline $\mathrm{Al}_{2} \mathrm{O}_{3}: \mathrm{C}$ & Harshaw Chemical & Single crystal \\
$(\mathrm{TLD} 500)$ & Co. USA & $\phi 5 \times 0.9$ \\
& & 72 \\
$\mathrm{LiF}: \mathrm{Mg}, \mathrm{Ti}$ & Harshaw Chemical & Chip \\
$(\mathrm{TLD} 700)$ & Co. USA & $4 \times 4 \times 2$ \\
& & 23.5 \\
$\mathrm{GeDi}$ & iXFiber SAS & Powder \\
Optical fiber & France & $0.0625 / 0.125$ \\
& & 49.2 \\
\hline
\end{tabular}

were originated from the same preform. The set is fabricated through Modified Chemical Vapor Deposition (MCVD) process by iXFiber SAS. The difference between these samples is due only to the drawing conditions. GeD1 was drawn with a speed of $70 \mathrm{~m} / \mathrm{min}, \mathrm{GeD} 2$ at $40 \mathrm{~m} / \mathrm{min}$ and GeD3 at $22 \mathrm{~m} / \mathrm{min}$. The Germanium (Ge) content along these fibers was specifically designed to follow a two-step distribution. The first step is doped with $\sim 4.5$ wt. $\%$ whereas the second is doped with $\sim 9$ wt. $\%$. The Ge doping profile has been already reported by Alessi et al. [9]-[11]. Such fibers allow us to investigate the influence brought out by varying the process changes in specialty fibers through the whole range of accessible drawing parameters.

For comparison, two off-the-shelf and commercially available TLDs (TLD700, TLD500) were used. They consist of LiF: $\mathrm{Mg}$, Ti chips and anion deficient $\mathrm{Al}_{2} \mathrm{O}_{3}$ single-crystals respectively. Their physical characteristics are summarized in Table I.

Due to heating associated to the TSL measurements, the polymer cladding of the fibers ( $250 \mu \mathrm{m}$ diameter) is removed over a length of 2 to $3 \mathrm{~m}$ before the analysis begin, also the fibers are being, with or without coating, during irradiation. The obtained bare fibers are cleaned with alcohol, and then cut in small pieces of few millimeters in length and finally placed on a $10 \mathrm{~mm}$ diameter aluminum pellet for TSL analysis.

\section{B. Irradiation Facilities}

All irradiations have been performed at room temperature (RT) and in air. X-ray irradiations were achieved by means of an X-ray source (XRG3500-Inel), available at LPMC, University of Nice. The $\mathrm{X}$-ray tube is composed by a $\mathrm{Cu}$ target with an accelerating voltage of $45 \mathrm{kV}$. Calibration of the beam is achieved by positioning the ionization chamber at the same distance as the studied fibers and TLDs from the Be window. The ionization chamber has a sensitive volume of $200 \mathrm{~mm}^{3}$ and is connected to a high precision/high resolution electrometer (PTW-France). $\mathrm{A}^{60} \mathrm{Co}$ gamma cell delivers $\gamma$ rays with different dose rates at TRAD's GAMRAY facility in Toulouse, France. Neutron irradiations with particles at two energy levels have been performed $(0.8$ and $14 \mathrm{MeV})$ using PROSPERO and SAMES facilities of CEA Valduc, France. $63 \mathrm{MeV}$ proton irradiations were performed at TRIUMF, Vancouver, Canada and at the Biomedical Cyclotron of Centre Antoine Lacassagne (CAL) in Nice, France.

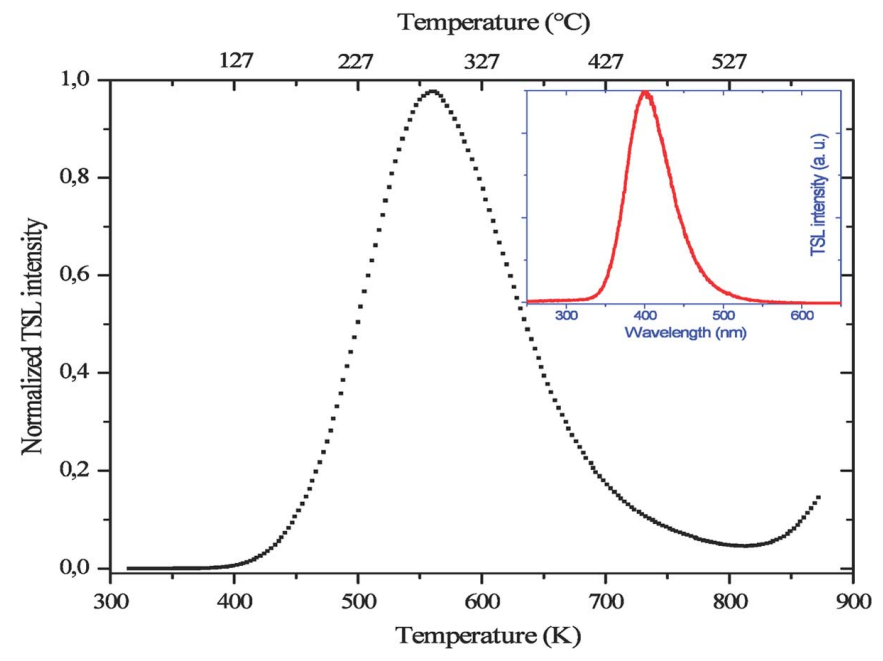

Fig. 1. Typical TSL curve of a Ge-doped optical fiber (here GeD2) after X-ray irradiation at RT.

\section{TSL Setup}

The TSL signal within temperature range from RT to 875 $\mathrm{K}$ was recorded with a linear heating rate of $2 \mathrm{~K} / \mathrm{s}$ by means of a photomultiplier tube (PMT). The emission integration is spanned over a $250-600 \mathrm{~nm}$ interval.

TSL emission spectra were detected by an optical multichannel analyzer (OMA), consisting of an optical fiber connected to a spectrograph equipped with a CCD array (Roper Scientific SAS). An appropriate grating centered around 500 $\mathrm{nm}$, covering the $300-800 \mathrm{~nm}$ range is used.

\section{RESULTS AND DisCUSSION}

The TSL curve of GeD2 fiber after X-ray irradiation is shown in Fig. 1. This typical curve is the single representation of all those measured fiber samples. It is composed of a broad, dominant peak located at $560 \mathrm{~K}$, which is extremely sensitive to the amount of absorbed dose and thus, well suited to be used in dosimetry field. This convenient position at relatively high temperature authorizes us to measure absorbed dose, both at room and high temperature especially in difficult-to-access areas, such as the "hermetic" zone in nuclear power plants. The trapping parameters (trap depth and frequency escape factor), which relate to this peak have already been determined elsewhere [12]. The insert in Fig. 1 shows a blue-violet luminescence band centered at $400 \mathrm{~nm}$, corresponding to the spectral distribution of this TSL peak. This emission falls in the middle of the spectral response of all UV-Vis PM tubes, and it can be easily extracted from the blackbody radiation of the TSL heater, and is thus very useful for dosimetry purposes. This emission is well known and has already been attributed to the recombination of released electrons on the two-fold $\mathrm{Ge}$ centers (= Ge :) [13].

In light of these findings, we decide first to analyze the behavior of these fibers to some basic extent of the required dosimetric criteria by comparing to two commonly used TLDs. For the reason of beam time availability, this comparison was made only for X-rays, which are available in the laboratory. Then, these promising results led us to investigate the TSL response 


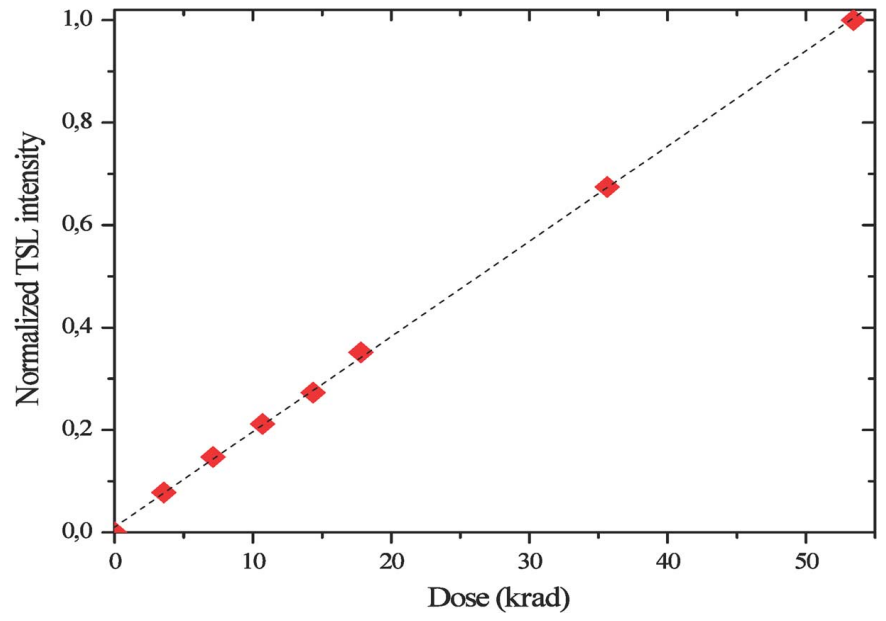

Fig. 2. TSL response of the dosimetric peak as a function of the X-ray absorbed dose for a dose-rate of $216 \mathrm{krad}(\mathrm{Si}) / \mathrm{h}$.

of GeDi $(i=1,2,3)$ fibers to various radiation sources $(\gamma$ photons, neutrons and protons).

\section{A. TSL Response Sensitivity and Dosimetric Criteria}

1) TSL Response to X-Ray Irradiation: During the analysis, up to more than $50 \mathrm{krad}$ of dose are applied. Fig. 2 shows that the dosimetric TSL peak response of all the three studied GeDi fibers (as well as the preforms) varies linearly with the absorbed dose (the observed correlation coefficient is better than 0,999). This might be particularly true at low dose levels (below $1 \mathrm{krad}$ ), which represent adequate dose range level for conventional radiation therapy. In addition, measurements at different dose rates do not show any dependence of the TSL response [12].

2) TSL Sensitivity: Ge-doped fibers which exhibit a very sensitive TSL response in Fig. 1 (by comparison to undoped or to F-, Al-, P- or RE-doped fibers [14] for which TSL curves are not reported here) are characterized by a very linear behavior (Fig. 2). Following these observations, we wanted to know how this optical fiber compared to two commercial TLDs by conducting a comparative study of the TSL response under the same conditions of irradiation and readout. The aim of this comparison is to test the ability of the Ge-doped fiber for medical radiation dosimetry at therapy levels in terms of sensitivity. The choice of X-rays $(45 \mathrm{keV})$ is due to their permanent availability in the laboratory and to their prominence at higher energy, along with electrons, in radiotherapy dosimetry.

Fig. 3 shows that under same conditions, GeD2 fiber, is at least, 10 times more sensitive than TLD500. In this figure, the normalized relative responses of both Ge-doped fibers and TLD500 are probably overestimated relative to that of TLD700. This particular observation might be due to varying effective atomic number $\left(\mathrm{Z}_{\mathrm{eff}}=11.9-13.4\right.$ for Ge-doped fibers [15], 10.2 for TLD500 [16], and 8.04 of TLD700 (LiF)). Indeed, at $45 \mathrm{keV}$, the relative response has a strong dependence on $\mathrm{Z}_{\text {eff }}$. However, due to lack of simulations, we cannot make an accurate estimation. The TLD is considered as "tissue equivalent" if it has a value of $\mathrm{Z}_{\text {eff }}$ near 7.4 of soft tissue. By way of comparison, it should be noted that the value of $\mathrm{Z}_{\text {eff }}$ for bones

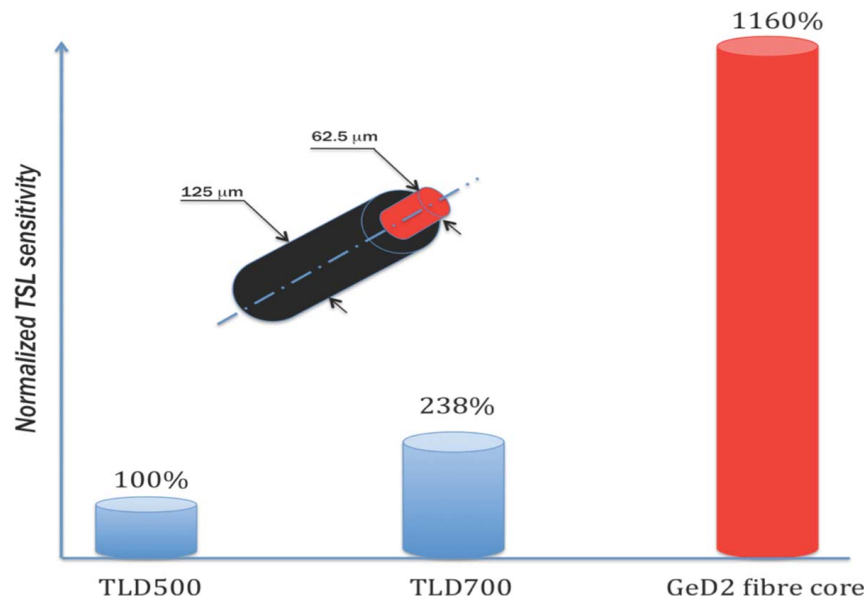

Fig. 3. Compared normalized TSL sensitivities of GeD2 fiber, TLD 500 and TLD700 dosimeters after X-ray irradiation at RT.

varies between 11.6 and 13.8 [16]. Thus, Ge-doped fibers in the lower energy range $(<200 \mathrm{keV})$ therefore require the use of appropriate filters for energy compensation as for $\mathrm{CaF}_{2}$ and $\mathrm{CaSO}_{4}$ TLD phosphors $\left(\mathrm{Z}_{\text {eff }}=16.3\right.$ and 14.4 respectively) [16] or reserve their use for the dosimetry of high-energy photon radiation (no $\mathrm{Z}_{\text {eff }}$ dependence) that is now widely used in radiotherapy centers.

3) Repeatability: each GeDi fiber was subjected to a test of repeatability consisting on 5 successive cycles (irradiation and readout). The result of this test was estimated to be less than $3 \%$ without any particular treatment while TLD700 chip is marketed with specification of $2 \%$ but only following a heavy thermal treatment $\left(1 \mathrm{~h}\right.$ at $400^{\circ} \mathrm{C}$ and $2 \mathrm{~h}$ at $\left.100^{\circ} \mathrm{C}\right)$ before each use. However, the reproducibility tests on different samples were not performed, but the results presented in the second part of this paper show that, for a given dose of radiation, even fibers of different diameters exhibit approximately the same TSL response.

4) Fading: Thermal fading is $5 \%, 8$ hours post irradiation and the optical fading is $6 \%$ after 6 hours but reaches $30 \%, 90$ hours post irradiation. Even though the latter percentage might appear striking, it is much lower than in the case of TLD500 for which the loss of information on the dose reaches $83 \%$ only after 10 minutes of light exposure [17].

5) Heating Rate Effect: Variation of the heating rate during TSL reading may induce a decrease of the response efficiency; a phenomenon particularly observed through TLD500 and known as thermal quenching of F centers in aluminum oxide [16] where the loss of TSL efficiency may reach $\sim 50 \%$ when the heating rate increases from 1 to $5 \mathrm{~K} / \mathrm{s}$. However, this effect is completely absent in the Ge-doped silica fibers since the TSL response remains the same when the heating rate varies from 1 to $10 \mathrm{~K} / \mathrm{s}$. This no heating rate dependence is still advantageous for dosimetry applications.

These data show that Ge-doped fiber is more sensitive than the two TLDs. The repeatability is within $3 \%$ without any usage specification, unlike TLD700, which requires heavy regeneration protocol $\left(1 \mathrm{~h}\right.$ at $400^{\circ} \mathrm{C}$ and $2 \mathrm{~h}$ at $\left.100^{\circ} \mathrm{C}\right)$ before each use, causing a waste of time especially in routine dosimetry. The thermal fading is low and the optical one is by far, better than 


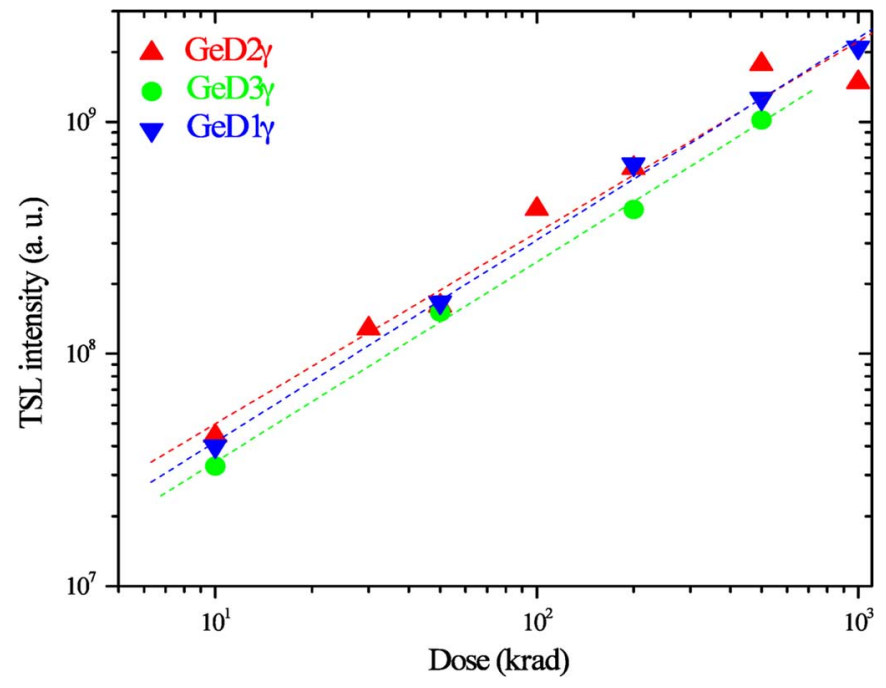

Fig. 4. TSL response as a function of the absorbed $\gamma$ dose at $500 \mathrm{rad}(\mathrm{Si}) / \mathrm{h}$ on the 3 fibers.

that of TLD500 and the thermal quenching effect is non-existent [12]. This entire set of significant results makes Ge-doped fiber a potential candidate in the field of dosimetry at therapy levels.

\section{B. TSL Response of GeDi Fibers to $\gamma, n$ and $p$ Radiations}

Beyond the clinical application of the fiber, we studied its behavior with respect to other sources of radiation.

1) Response to ${ }^{60} \mathrm{Co} \gamma$-Rays: The TSL response as a function of the $\gamma$ - absorbed dose, deposited at a dose rate of 500 $\operatorname{rad}(\mathrm{Si}) / \mathrm{h}$, is illustrated in Fig. 4. It shows a linear behavior within a wide range of total dose for each of the three fibers.

The same set of measurements has also been performed at $\gamma$ dose rates of 30 and $100 \mathrm{rad}(\mathrm{Si}) / \mathrm{h}$ and both lead to the same TSL response behaviors than that presented in Fig. 4. One can conclude that within this range of doses and dose rates, the dose rate produces no effect whatsoever. This statement is in good agreement with the results of Mady et al. [18] showing that no clear dose rate dependence is established in these same Ge-doped fibers.

The linear behavior observed, in up to $50 \mathrm{krad}(\mathrm{Si})$ for X-ray irradiation (Fig. 2) is largely confirmed since no saturation behavior is observed even after $\gamma$-rays doses are increased to al$\operatorname{most} 1 \mathrm{Mrad}(\mathrm{Si})$.

More generally, Figs. 2 and 4 show that the TSL response is proportional to the Total Ionizing Dose (TID) since it corresponds to the portion of energy absorbed by the fibers during the whole exposure.

2) Response to Neutrons: Contrary to X-rays, which interact dominantly with the electron shells of the atom, neutrons are electrically neutral and interact with the nucleus of the target atom. They are still capable of ionizing atoms through different indirect reactions. The TSL measurements, after neutron irradiations, exhibit quite similar responses to those obtained for $\gamma$ - or X-rays irradiation whose typical glow curve is shown in Fig. 1, with corresponding neutron energies of $0.8 \mathrm{MeV}$ and 14 $\mathrm{MeV}$. In addition to its performance as a TL dosimeter already

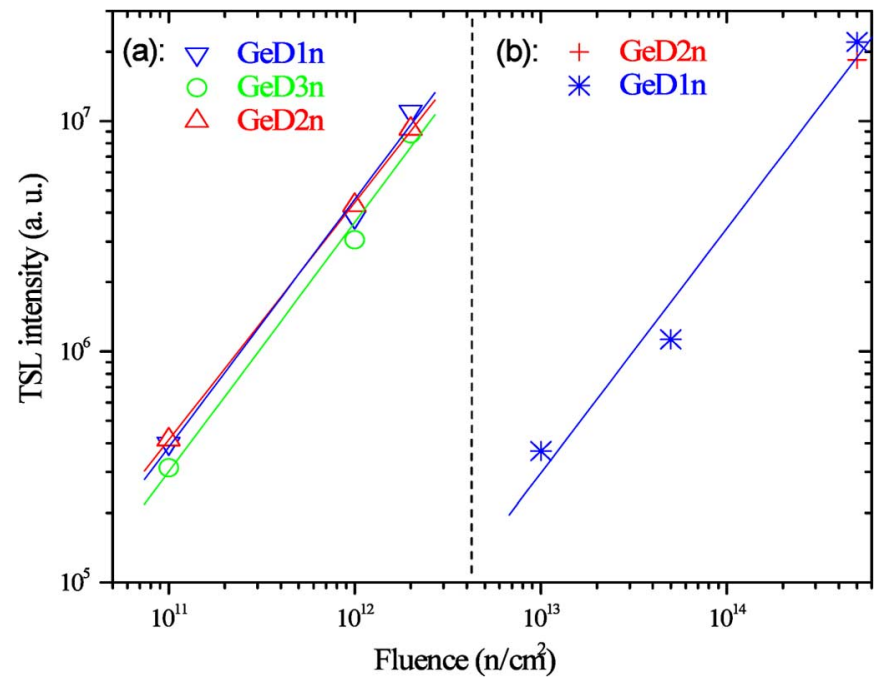

Fig. 5. TSL response as a function of neutron fluence for GeD1, GeD2 and GeD3 fibers after RT irradiation at energies of: $14 \mathrm{MeV}$ (a) and $0.8 \mathrm{MeV}$ (b).

outlined in part A of this section, the Ge-doped fiber is also sensitive to neutrons. The corresponding evolution of the TSL intensity of GeDi optical fibers as a function of neutron fluence at energies of 14 and $0.8 \mathrm{MeV}$, is shown in Fig. 5.

Another additional advantage of the fiber-based dosimeter, compared to both commercial TLD, is the neutron sensitivity. It is because, in a neutron environment, TLD700 becomes sensitive only when it is enriched with ${ }^{7} \mathrm{Li}$ isotope. In the meantime the TLD500 exhibits no sensitivity at all.

Fig. 5 shows that, for $14 \mathrm{MeV}$ neutrons, all three fibers present almost identical TSL intensities and exhibit a similar linear behavior as a function of fluence. Two of these fibers were also tested at energy of $0.8 \mathrm{MeV}$, but in this case, there is not enough data to conclude satisfactorily. This is probably due to the fact that for this reactor irradiation, we plot the results by considering a monoenergetic irradiation at $0.8 \mathrm{MeV}$ whereas the fibers are in fact, exposed to fission neutrons covering a larger range of energies. Morin et al. have presented a detailed description of PROSPERO facility [19].

3) Response to Protons: In Fig. 6, the variations of the TSL response versus $63 \mathrm{MeV}$ proton fluence for the three $\mathrm{GeDi}$ fibers, are reported. These data confirm that the fibers are also sensitive to protons. Moreover, between $3 \times 10^{10}$ and $3 \times 10^{11}$ $\mathrm{p} / \mathrm{cm}^{2}$, the TSL intensity is directly proportional to the particle fluence.

4) The Overall Response as a Function of the Ionizing Dose: The glow curves obtained under different types of radiations $(\gamma$ - rays, neutrons and protons) are all similar in shape to that observed after X-ray irradiation (Fig. 1), i.e., a broad peak around $550 \mathrm{~K}$ is observed, with a full width at half maximum (FWHM) exceeding $100 \mathrm{~K}$. The corresponding luminescence of this peak is the band around $400 \mathrm{~nm}$ (shown in insert of Fig. 1).

This similarity between all the TSL curves whatever the irradiation is, means that for the moderate fluences used in this work, the defects generated in the fibers are mostly induced by ionization and not by displacement damage. This is in good agreement with the results obtained by Girard et al. [20], showing that the Radiation Induced Attenuation (RIA), thus 


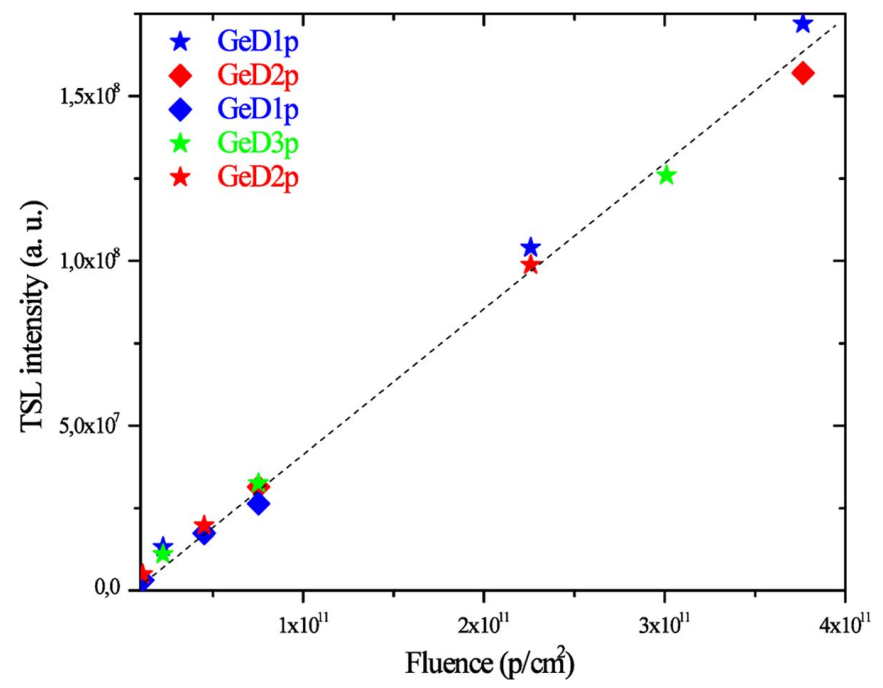

Fig. 6. TSL response as a function of proton fluence for GeD1, GeD2 and GeD3 fibers after irradiation at RT ( $\star$ : Cyclotron, $\downarrow$ : Triumf).

the defect density, is similar for $\mathrm{X}-, \gamma$-rays and $14 \mathrm{MeV}$ neutron irradiations in different fibers, including germanosilicate optical fibers.

Then, using the well-known models, providing ionizing dose as a function of the fluence for each type of particle at a given energy, the following conversions of particle fluence to $1 \mathrm{rad}(\mathrm{Si})$ of indirect ionizing dose were performed [21]-[23]:

$-7.53 \times 10^{6} \mathrm{p} / \mathrm{cm}^{2}$ of $63 \mathrm{MeV}$ protons

$-1.2 \times 10^{9} \mathrm{n} / \mathrm{cm}^{2}$ of $14 \mathrm{MeV}$ neutrons and

$-2.6 \times 10^{11} \mathrm{n} / \mathrm{cm}^{2}$ for neutrons of $0.8 \mathrm{MeV}$

Considering these conversion factors, a new TSL response curve as a function of direct or indirect ionizing dose is presented in Fig. 7, compiling the results of all our irradiation tests. These results showed that the generation of the defects related to the monitored TSL response are due to direct ionization for photons and indirect ionization for protons and neutrons. In that sense, the behavior of amorphous silica and silicon under neutron irradiation are quite different at the tested fluence levels $\left(<10^{15} \mathrm{n} / \mathrm{cm}^{2}\right)$, as clear evidences exist for distinct TID and DDD-related defects in Si [24]. This "universal" response includes all the data shown in Figs. 4-6, corresponds respectively to $\gamma$-rays, neutrons of 0.8 and $14 \mathrm{MeV}$ and $63 \mathrm{MeV}$ protons, and this is for the three fibers drawn differently from the same preform.

\section{CONCLUSION}

The results show that Ge doped silica-based optical fiber is very attractive for TSL dosimetry applications. On the TSL glow curve, the position of the dosimetric peak storing the information on the absorbed dose, is located almost perfectly: high enough to enable measurement of the absorbed dose at high temperature, and low enough to allow a complete determination of the absorbed dose. This property will open the way for such dosimeters to be used in harsh environments, combining high temperature and difficult-to-access areas, such as that of nuclear power plants for example.

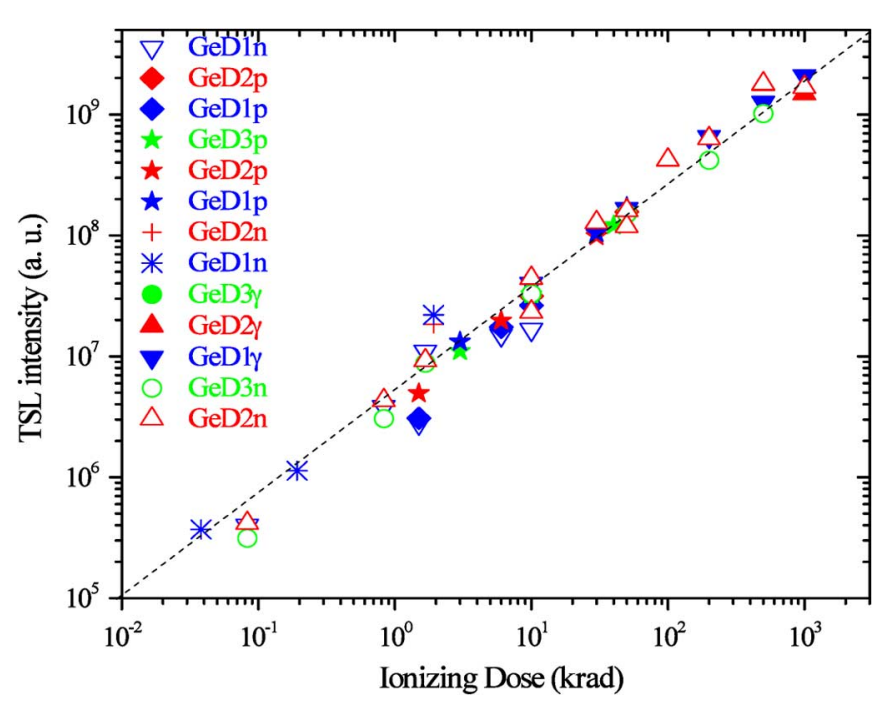

Fig. 7. TSL response as a function of "direct or indirect" ionizing dose for $\mathrm{GeD} 1, \mathrm{GeD} 2$ and GeD3 fibers after $\gamma$-rays, neutron $(14$ and $0.8 \mathrm{MeV})$ and 63 $\mathrm{MeV}$ proton irradiations.

At therapy levels, the Ge-doped fiber meets all the main criteria required by the clinical dosimetry, often offers better features than the two commercial TLDs even when comparison is drawn on their own ground operation. It has good response linearity to photons and does not show any dose rate dependence, which may be useful for physicists.

The response shows a good neutron- and proton- detection efficiency and high gamma ray sensitivity.

In addition, the Ge-doped fiber allows quantifying the fluences of monoenergetic radiations of both neutrons and protons, but can probably also be used in the case of neutron spectra if their energy distribution is known.

All of these highly desirable features open the way to a "universal" dosimeter, which is particularly interesting, not only at therapy levels but also for the dosimetry associated with radiation test facilities, such as those providing low energy protons for SEE measurements.

The results show that for a given dose (or fluence), the TSL response is almost the same on the three fibers. So there is no drawing conditions dependency. This will probably further reduce the cost of the already less expensive mentioned fiber, which is potentially much cheaper than the TLDs with which it was compared to.

In addition to all these advantages, the dimension of the optical fiber is intrinsically smaller than that of TLDs, and can be exploited for dosimetry applications in areas with high gradients of dose.

Work is still ongoing on this new type of dosimeter, in order to expand the particle energy range in which the linearity of the TSL response versus fluence (or ionizing dose) is experimentally observed.

\section{ACKNOWLEDGMENT}

The authors would like to thank J. Hérault and G. Angelier of Centre Antoine Lacassagne (CAL) Biomedical Cyclotron in 
Nice for their assistance and technical help during proton irradiations and the TRIUMF's committee for providing the proton beam time.

\section{REFERENCES}

[1] A. Alessi, S. Girard, M. Cannas, S. Agnello, A. Boukenter, and Y. Ouerdane, "Evolution of photo-induced defects in Ge-doped fiber/preform: Influence of the drawing," Opt. Exp., vol. 19, no. 12, pp. 11680-11690, 2011.

[2] A. Alessi, S. Agnello, S. Grandi, A. Parlato, and F. M. Gelardi, "Refractive index change dependence on $\mathrm{Ge}(1)$ defects in $\gamma$-irradiated Ge-doped silica," Phys. Rev. B, vol. 80, pp. 0141031-0141036, 2009.

[3] M. Fujimaki, T. Katoh, T. Kasahara, and Y. Ohki, "Paramagnetic centres induced in Ge-doped silica glass with UV irradiation," J. Phys. Condens. Matter, vol. 11, pp. 2589-2594, 1999.

[4] E. J. Friebele, D. L. Griscom, and G. H. Sigel, "Defect centers in a germanium-doped silica-core optical fiber," J. Appl. Phys., vol. 45, no. 8, pp. 3424-3428, 1974.

[5] A. Youssef, A. Abdulla, Y. M. Amin, and D. A. Bradley, "The thermoluminescence response of Ge-doped optical fibre subjected to photon irradiation," Radiat. Phys. Chem., vol. 61, pp. 409-410, 2001.

[6] S. Hashim, S. Al-Ahbabi, D. A. Bradley, M. Webb, C. Jeynes, A. T Ramli, and H. Wagiran, "The thermoluminescence response of doped $\mathrm{SiO}_{2}$ optical fibres subjected to photon and electron irradiations," Appl. Radiat. Isot., vol. 67, pp. 423-427, 2009.

[7] A. T. Abdul Rahman, R. P. Hugtenberg, S. F. Abdul Sani, A. I. M. Alalawi, F. Issa, R. Thomas, M. A. Barry, A. Nisbet, and D. A. Bradley, "An investigation of the thermoluminescence of Ge-doped $\mathrm{SiO}_{2}$ optical fibres for application in interface radiation dosimetry," Appl. Radiat. Isot., vol. 70, no. 6, pp. 1436-1441, 2012.

[8] S. Girard, J. Kuhnhenn, A. Gusarov, M. Van Uffelen, Y. Ouerdane, A. Boukenter, and C. Marcandella, "Radiation effects on silica-based optical fibers: Recent advances and future challenges," IEEE Trans. Nucl. Sci., vol. 60, no. 3, pp. 2015-2036, Jun. 2013.

[9] A. Alessi, S. Girard, C. Marcandella, S. Agnello, M. Cannas, A. Boukenter, and Y. Ouerdane, "X-ray irradiation effects on a multistep Ge-doped silica fiber produced using different drawing conditions," $J$. Non-Cryst. Solids, vol. 357, pp. 1966-1970, 2011.

[10] S. Girard, Y. Ouerdane, G. Origlio, C. Marcandella, A. Boukenter, N. Richard, J. Baggio, P. Paillet, M. Cannas, J. Bisutti, J. P. Meunier, and R. Boscaino, "Radiation effects on silica-based performs and optical fibers: Experimental study with canonical samples," IEEE Trans. Nucl. Sci., vol. 55, no. 6, pp. 3473-3482, Dec. 2008.
[11] A. Alessi, S. Girard, C. Marcandella, M. Cannas, A. Boukenter, and Y. Ouerdane, "Micro-raman investigation of $\mathrm{X}$ or $\gamma$ irradiated Ge doped fibers," Nucl. Instr. Meth. Phys. Res. B, vol. 269, pp. 1346-1349, 2011

[12] M. Benabdessselam, F. Mady, and S. Girard, "Assessment of Ge-doped optical fibre as a TL-mode detector," J. Non-Cryst. Solids, vol. 360, pp. 9-12, 2013.

[13] L. Skuja, G. Pacchioni, L. Skuja, and D. L. Griscom, Eds., "Optical properties of silica," Defects in $\mathrm{SiO}_{2}$ and Related Dielectrics: Science and Technology p. 108, 2000.

[14] M. Benabdesselam, F. Mady, and S. Girard, "Assessment of Ge-doped optical fibre as a TL-mode detector," in Proc. 9th Symp. SiO ${ }_{2}$ Advanced Dielectrics and Related Devices, Hyères, France, Jun. 17-20, 2012, pp. $51-52$.

[15] H. Wajiran, I. Hossain, H. Asni, and A. T. Ramli, "Thermoluminescence energy response of a germanium-doped optical fiber obtained using a monte carlo N-particle code simulation," J. Kor. Phys. Soc., vol. 59, no. 2, pp. 337-340, Aug. 2011

[16] S. McKeever, Thermoluminescence of Solids. Cambridge, U.K. Cambridge Univ. Press, 2005.

[17] M. Moscovitch, R. A. Tawil, and M. Svinkin, "Light induced fading in $\alpha-\mathrm{Al}_{2} \mathrm{O}_{3}$ : C," Radiat. Prot. Dosim., vol. 47, pp. 251-253, 1993.

[18] F. Mady, M. Benabdesselam, J. B. Duchez, Y. Mebrouk, and S. Girard, "Global view on dose rate effects in silica-based fibers and devices damaged by radiation-induced carrier trapping," presented at the 50th NSREC, San Francisco, CA, USA, Jul. 8-12, 2013.

[19] J. Morin and P. Zyromski, "The PROSPERO and CALIBAN neutron irradiation facilities," in Proc. RADECS91, La Grande Motte, France, 1991, pp. 574-576.

[20] S. Girard, J. Baggio, and J. Bisutti, "14-MeV neutron, $\gamma$-ray, and pulsed $\mathrm{x}$-ray radiation-induced effects on multimode silica-based optical fibers "," IEEE Trans. Nucl. Sci., vol. 56, no. 6, pp. 3750-3757, Dec. 2006.

[21] V. A. J. Van Lint, G. Gigas, and J. Barengolt, "Correlation of displacement effects produced by eectrons, protons and neutrons," IEEE Trans. Nucl. Sci., vol. 22, no. 6, pp. 2663-3757, Dec. 1975.

[22] V. A. J. Van Lint, T. M. Flanagan, R. E. Leadon, J. A. Naber, and V. C. Rodgers, Mechanisms of Radiation Effects in Electronic Materials. New York, NY, USA: Wiley, 1980, vol. 1.

[23] J. L. Leray, "Total dose effects: Modelling," in IEEE Nuclear and Space Radiation Effects Conference Short Course, 1999, NSREC Short Course Notebook, in CDROM available at the IEEE Library.

[24] J. R. Srour and J. W. Palko, "A framework for understanding displacement damage mechanisms in irradiated silicon devices," IEEE Trans. Nucl. Sci., vol. 53, no. 6, pp. 3610-3620, Dec. 2006. 